W.Gorski et al.

\title{
Electrochemical Sensing Based on Redox Mediation at Carbon Nanotubes
}

Supporting information (5 Figures).

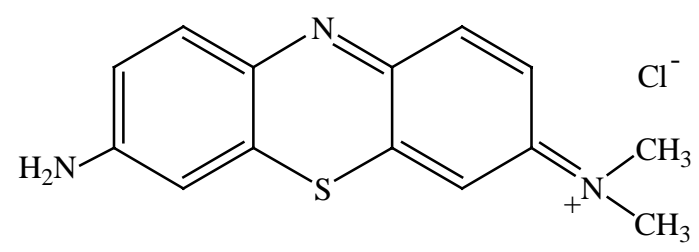

(a) Azure A

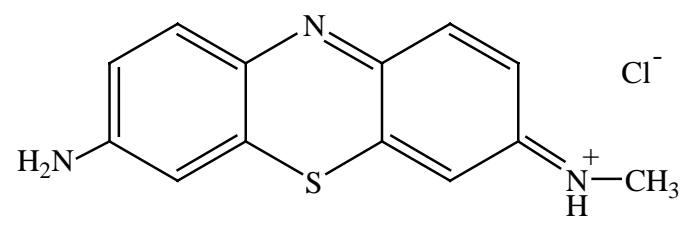

(b) Azure C

Figure S1. Chemical structures of Azure dyes. 
W.Gorski et al.

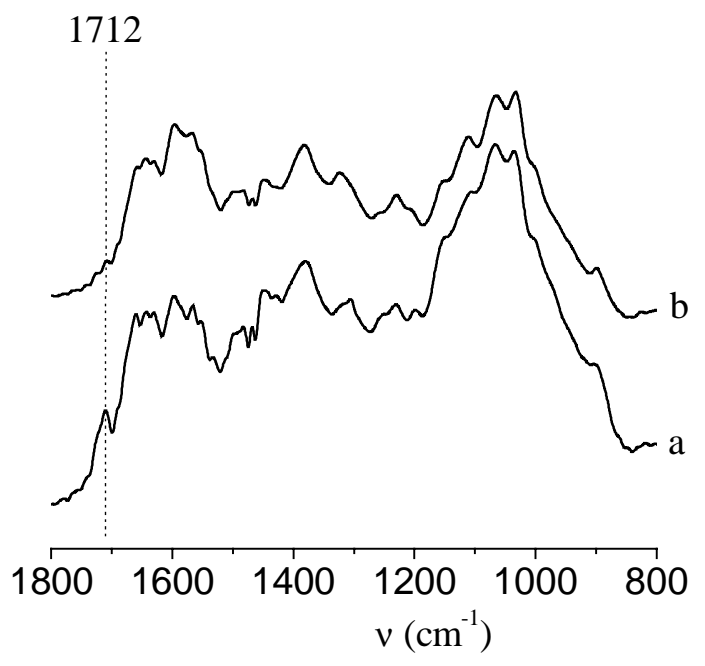

Figure S2. Infrared spectra of films (a) CHIT-AZU, and (b) reduced CHIT-AZU using sodium cyanoborohydride. The reduction eliminated the absorption band of aldehyde at $1712 \mathrm{~cm}^{-1}$.

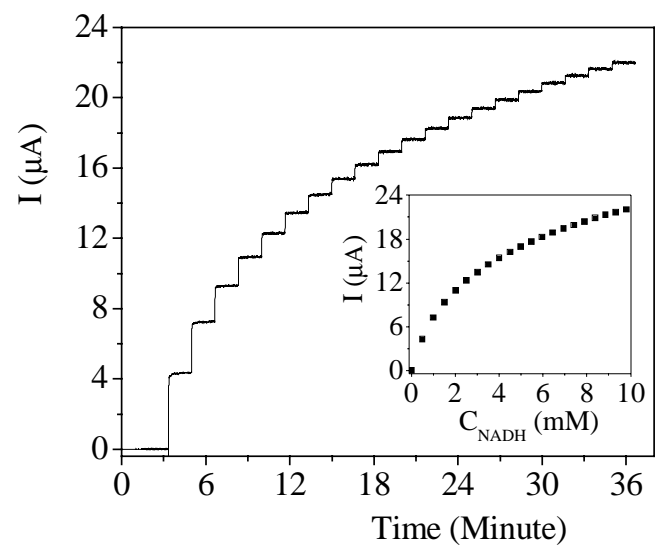

Figure S3. Amperometric response $(\mathrm{E}=-0.10 \mathrm{~V})$ of a CHIT-AZU/CNT film electrode to additions of $0.50 \mathrm{mM}$ NADH aliquots, which illustrated a wide dynamic range up to $10 \mathrm{mM}$ NADH. Inset: a relevant calibration plot. Background electrolyte, stirred phosphate buffer ( $\mathrm{pH}$ 7.40). 
W.Gorski et al.

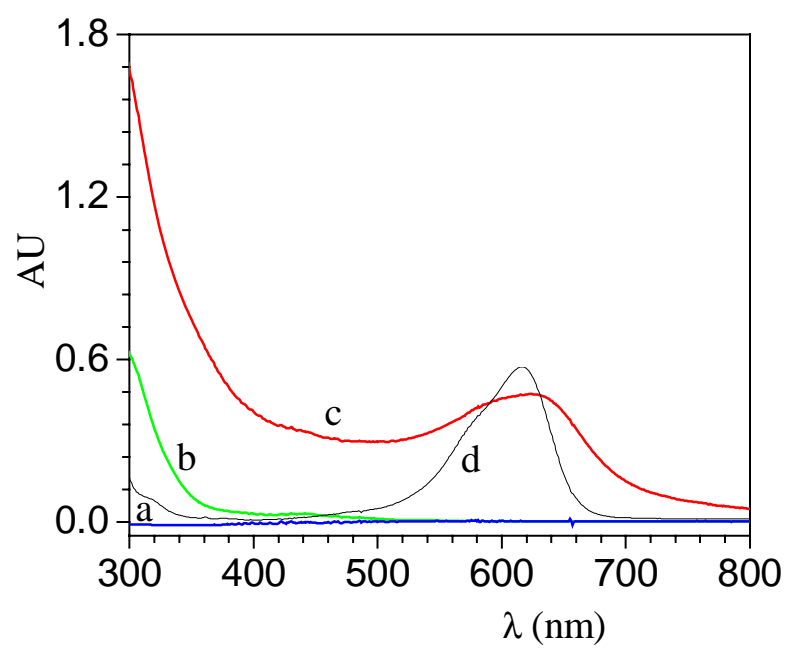

Figure S4. UV-visible spectra of 0.01 wt. \% solutions of (a) CHIT, (b) CHIT-glutaric dialdehyde, and (c) CHIT-AZU. Spectrum (d) is for $5.0 \mu \mathrm{M}$ solution of free AZU. Solutions b and c correspond to solutions I and II, respectively, according to labeling used in the paper.

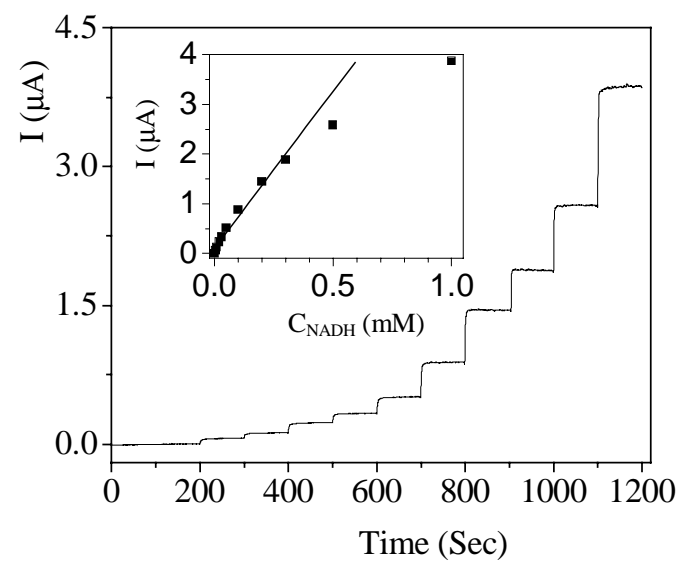

Figure S5. Amperometric response $(\mathrm{E}=-0.10 \mathrm{~V})$ of a CHIT-AzureA/CNT film electrode to additions of NADH aliquots $(5.0 \mu \mathrm{M}-1.0 \mathrm{mM})$ into a stirred solution of phosphate buffer, $\mathrm{pH} 7.40$. Inset: a calibration plot. 
W.Gorski et al.

Sensitivity of CHIT-AzureA/CNT electrodes was equal to $\sim 6.3 \mathrm{~mA} \mathrm{M}{ }^{-1}(1.5 \mu \mathrm{M}-0.30 \mathrm{mM}$, $\mathrm{R}^{2}=0.990$ ), which was $\sim 60 \%$ of the sensitivity displayed by the CHIT-AZU/CNT electrodes (AZU = Azure C). 